283

SPORTS INJURY PREVENTION PROGRAMS: AWARENESS, IMPLEMENTATION AND OPINION OF PHYSICAL THERAPISTS WORLDWIDE

\begin{abstract}
1,2,3Wesam Saleh A Al Attar, 'Sameer A Yamani, 'Eyad S Alharbi, 'Majed M Aljabri, ${ }^{4}$ Hussain Saleh H Ghulam, ${ }^{5}$ Saud Alarifi, ${ }^{3}$ Ross H Sanders. ${ }^{1}$ Department of Physical Therapy, Faculty of Applied Medical Science, Umm Al Qura University, Makkah, Saudi Arabia; ${ }^{2}$ Department of Sport, Exercise and Health, Faculty of Medicine, University of Basel, Basel, Switzerland; ${ }^{3}$ Discipline of Exercise and Sport Science, Faculty of Medicine and Health, The University of Sydney, Sydney, Australia; ${ }^{4}$ Department of Rehabilitation Medical Sciences, Faculty of Applied Medical Science, Najran University, Najran, Saudi Arabia; ${ }^{5}$ Department of Physical Therapy, King Abdulaziz Medical City, Riyadh, Saudi Arabia
\end{abstract}

\subsection{6/bjsports-2021-IOC.261}

Background Injury prevention programs have been shown to prevent and reduce injuries in different sports. Awareness for such programs is crucial, however, implementation and adoption of injury prevention programs in team sports is a multifaceted and complex process.

Objective To assess physical therapists' awareness, implementation, and opinion of sports injury prevention programs, from an international perspective.

Design A cross-sectional study.

Setting Online survey.

Patients (or Participants) A total of 484 male (44.4\%) and female $(55.6 \%)$ physical therapists participated in the study. Interventions (or Assessment of Risk Factors) The questionnaire consisted of questions covering the awareness, implementation, and physical therapists' opinion of different injury prevention program such as: (The FIFA $11+, 11+$ Kids, $11+$ Referees, 11 + Goalkeepers, GAA15, PEP, KIPP, Boksmart Rugby, Knäkontroll, iSPRINT and ACC SportSmart injury prevention programs). Main Outcome Measurements The study primary outcomes were awareness level, implementation rate, and opinion of the effectiveness of the sports injury prevention programs.

Results A total of 287 (59.3\%) of the participants were aware of the current sports injury prevention programs, 177 (36.6\%) were implementing sports injury prevention programs in their current practice. Participants who implemented the sports injury prevention programs reported a positive opinion about the program efficacy, with a score of $7.3 \pm 2.1$ out of 10 .

Conclusions In order to increase the awareness and implementation of sports injury prevention program, further work needs to be done to educate physical therapists about the importance of implementing sports injury prevention programs and the effectiveness in preventing injuries.

\section{THE EFFECT OF THE FÉDÉRATION INTERNATIONALE DE FOOTBALL ASSOCIATION (FIFA) 11+ REFEREES INJURY PREVENTION PROGRAM IN REDUCING INJURY RATES AMONG SOCCER REFEREES AND ASSISTANT REFEREES: A RANDOMIZED CONTROLLED TRIAL}

\footnotetext{
1,2,3 Wesam Saleh A Al Attar, ${ }^{1}$ Fahad M Alkabkabi, ${ }^{1}$ Nasser G Alshamrani, ${ }^{4}$ Saud Alarifi, ${ }^{5}$ Hussain Saleh H Ghulam, ${ }^{6}$ Hosam Alzahrani, ${ }^{1}$ Eman F Aljedaani, ${ }^{3}$ Ross H Sanders. ${ }^{1}$ Department of Physical Therapy, Faculty of Applied Medical Science, Umm Al Qura University, Makkah, Saudi Arabia; ${ }^{2}$ Department of Sport, Exercise and Health, Faculty of Medicine, University of Basel, Basel, Switzerland; ' ${ }^{3}$ Discipline of Exercise and Sport Science, Faculty of Medicine and Health, The University of Sydney, Sydney, Australia; ${ }^{4}$ Department of Physical Therapy, King Abdulaziz Medical City, Riyadh, Saudi Arabia; ${ }^{5}$ Department of Rehabilitation Medical Sciences, Faculty of Applied Medical Science, Najran University, Najran, Saudi Arabia; ${ }^{6}$ Department of Physiotherapy, College of Applied Medical Sciences, Taif University, Taif, Saudi Arabia
}

10.1136/bjsports-2021-IOC.262
Background The Fédération International de Football Association (FIFA) $11+$ Referees injury prevention program is a structured warm-up program specially designed to prevent injuries among soccer referees and assistant referees and its efficacy is yet to be fully documented in the literature.

Objective The purpose of this study was to investigate the effectiveness of the FIFA $11+$ Referees injury prevention program in reducing injury rates among soccer referees and assistant referees.

Design Randomized controlled trial.

Setting Competitive soccer.

Patients (or Participants) Two hundred male soccer referees and assistant referees aged (32 4.24$)$, participated in this study. The participants were randomly allocated into the experimental or a control group.

Interventions (or Assessment of Risk Factors) The experimental group performed the FIFA $11+$ program as warm-up during training sessions for at least twice a week, and the control group performed their usual warm-ups. Participants were prospectively followed during one season (6 months).

Main Outcome Measurements The primary outcome was to investigate the incidence of initial, recurrent injury and injury severity. The secondary outcome was to measure the rate of compliance by a review of the registered participations.

Results A total of 24 injuries were reported among 100 referees in the control group in $16,606 \mathrm{~h}$ of exposure (1.45 injuries $/ 1000 \mathrm{~h}$ ), and a total of 9 injuries were reported across 100 referees within the experimental group in 17,834 exposure hours (0.50 injuries/1000 h). The injury risk ratio IRR was 0.35 (95\% CI 0.26 to 0.45$)$, which indicates that the injuries in the experimental group reduced by $65 \%$ when compared to the control group.

Conclusions Implementation of the FIFA $11+$ referees injury prevention program reduced overall injury rates in male soccer referees and assistant referees more than the usual warmup.

\section{CHARACTERIZING INJURY PREVENTATIVE BEHAVIOR: A SPORT PSYCHOLOGY PERSPECTIVE}

James Parker, Urban Johnson, Andreas Ivarsson. Halmstad University, Halmstad, Sweden

\subsection{6/bjsports-2021-IOC.263}

Background Sustainable injury preventative behavior is a key factor in increasing the duration an athlete is free from injury. A better understanding of the interplay between different psychological factors and injury preventative behavior (IPB) is needed to be able to translate current knowledge to real-life injury prevention.

Objective The aim of this study is to identify profiles that characterize self-determined motivation, intention of IPB, and enacted IPB among high-level athletes.

Design Cross-sectional descriptive study.

Setting On-line data collection.

Participants In total 90 high-level athletes gave consent and 29 golfers and 22 volleyball players completed all questionnaires $(21.6 \pm 3.8$ years, male $=30$, female $=21$, level: regional 5 , national 30, international 16)

Assessment of Risk Factors The OSTRC Questionnaire for overuse injury was used to collect data on injury severity. Selfdetermined motivation (SDT) and planned and enacted injury 